\title{
Egalitarian recruitment of localized mRNAs
}

\author{
Dmitry Nashchekin and Daniel St Johnston ${ }^{1}$ \\ The Gurdon Institute and the Department of Genetics, University of Cambridge, Cambridge CB2 1QR, United Kingdom
}

\begin{abstract}
Bicaudal-D (Bic-D) and Egalitarian (Egl) are required for the dynein-dependent localization of many mRNAs in Drosophila, but the mRNAs show no obvious sequence similarities, and the RNA-binding proteins that recognize them and link them to dynein are not known. In this issue of Genes \& Development, Dienstbier and colleagues (pp. 1546-1558) present evidence that the elusive RNA-binding protein is Egl itself. As well as linking mRNA to dynein, they show that Egl also activates dynein motility by binding Bic-D and the dynein light chain.
\end{abstract}

It is becoming increasingly clear that mRNA localization is a widespread mechanism for targeting proteins to the regions of a cell where they are required (St Johnston 2005; Martin and Ephrussi 2009). For example, a recent study found that up to $70 \%$ of mRNAs in early Drosophila embryos show discrete subcellular localizations (Lecuyer et al. 2007). Localizing mRNA increases the efficiency of protein targeting, and is particularly important when it would be deleterious to express the encoded protein elsewhere in the cell. This is the case for many key developmental determinants, which must therefore be localized as mRNAs. These include budding yeast Ash1p, which controls the asymmetric inheritance of mating-type switching; the Drosophila axis determinants Bicoid, Oskar, Nanos, and Gurken; and the Xenopus vegetal determinants Vg1 and VegT (Johnstone and Lasko 2001; King et al. 2005; Bastock and St Johnston 2008; Paquin and Chartrand 2008). Another advantage of mRNA localization is that it allows the synthesis of specific proteins to be locally regulated, and this is particularly important in large complex cells like neurons, where the control of translation is thought to play an important role in axon guidance and synaptic plasticity (Martin and Zukin 2006; Lin and Holt 2007).

Given the significance of mRNA localization, a great deal of effort has been directed toward understanding the underlying mechanisms. In many cases, mRNAs are

[Keywords: Drosophila; dynein; mRNA localization; microtubule-based transport; stem-loop]

${ }^{1}$ Correspohnding author.

E-MAIL d.stjohnston@gurdon.cam.ac.uk; FAX 44-1223-334-089.

Article is online at http://www.genesdev.org/cgi/doi/10.1101/gad.1821509. actively transported along actin filaments or microtubules (MTs) to their destinations by myosin, kinesin, or dynein motors. These mRNAs must therefore contain cis-acting localization elements that are recognized by RNA-binding proteins that link them to the appropriate motor. Many RNA-binding proteins that recognize mRNA localization signals have been identified, but how these link to the transport machinery has remained elusive. Indeed, the only case where the coupling of an mRNA to the motor has been elucidated is Ash1 mRNA, which is linked to the myosin Myo4p by the RNA-binding protein She2p and the myosin cargo adaptor protein She3p (Bohl et al. 2000; Gonsalvez et al. 2005).

\section{The dynein-dependent mRNA transport pathway}

Although there appear to be a wide variety of mRNA localization pathways, a large class of mRNAs localize to the apical cytoplasm of the Drosophila syncitial blastoderm embryo, including the mRNAs for pair-rule genes fushi tarazu (ftz), hairy (h), runt (run), and wingless mRNA (Davis and Ish-Horowicz 1991; Simmonds et al. 2001). At this stage of embryogenesis, the blastoderm nuclei form a monolayer beneath the apical cortex, and apical centrosomes nucleate MTs that grow around the nuclei with their plus ends extending into the basal cytoplasm. Since the pair-rule genes all encode transcription factors, the localization of their mRNAs close to the nuclei increases the nuclear concentration of pair-rule proteins and prevents their diffusion to other regions (Bullock et al. 2004). Wingless, on the other hand, is a signaling molecule, and the apical localization of its mRNA is necessary for the secretion of functional $\mathrm{Wg}$ protein (Simmonds et al. 2001). Injections of fluorescently labeled RNAs into syncitial blastoderm embryos have revealed that pair-rule and wingless transcripts are localized by active transport: The RNAs are rapidly incorporated into particles in the basal cytoplasm and then move in a dynein-dependent manner along MTs at speeds of up to $0.5 \mu \mathrm{m} / \mathrm{sec}$ toward the MT minus ends in the apical cytoplasm (Bullock and Ish-Horowicz 2001; Wilkie and Davis 2001).

Another mRNA transport pathway that is used by many different transcripts is the movement of mRNAs from the nurse cells of the Drosophila egg chamber into the oocyte. The oocyte nucleus is transcriptionally silent, 
and the transcripts for the localized determinants that define the body axes of the future embryo $(b c d, o s k, g r k$, and nos) must therefore be expressed in the nurse cells and transported through the ring canals into the oocyte before being localized to their specific destinations at the anterior, posterior, and dorsal regions of the oocyte (St Johnston et al. 1989; Forrest and Gavis 2003; Caceres and Nilson 2005; Jenny et al. 2006). A number of other transcripts use the same pathway (e.g., K10, nanos, and orbl, and a survey of randomly selected cDNAs suggested that $\sim 10 \%$ of mRNAs are transported from the nurse cells into the oocyte (Dubowy and Macdonald 1998). Like apical localization in the embryo, this nurse cell to oocyte movement is an active process in which the mRNAs move in a dynein-dependent manner toward the MT minus ends (Clark et al. 2007; Mische et al. 2007). Once the mRNAs enter the oocyte, they are then targeted to different destinations. grk, $b c d$, and $K 10$ still use dynein as the motor for transport and are localized to the anterior or dorsal/anterior of the oocyte, whereas osk and nanos mRNAs go to the posterior in a kinesin-dependent (osk) or motor-independent (nanos) fashion (Brendza et al. 2000; Forrest and Gavis 2003; MacDougall et al. 2003; Weil et al. 2006; Zimyanin et al. 2008).

Interestingly, oocyte-specific transcripts can be recognized by blastoderm localization machinery and vice versa (Bullock and Ish-Horowicz 2001). grk, bcd, K10, and nos RNAs accumulate in the apical cytoplasm after injection into a blastoderm embryo. Moreover, when $f t z$ and $h$ RNAs are ectopically expressed during oogenesis, they accumulate at the anterior of the oocyte. Thus, the nurse cell to oocyte pathway and the apical blastoderm pathway recognize the same localization signals and use a common machinery. Furthermore, it seems likely that the same pathway localizes inscuteable mRNA in embryonic neuroblasts and crumbs and stardust mRNAs in epithelial cells (Hughes et al. 2004; Horne-Badovinac and Bilder 2008; Li et al. 2008)

\section{Cis-acting signals for the dynein mRNA transport pathway}

Only a few of the RNA localization elements that direct dynein-dependent transport have been characterized so far. Two oocyte-specific transcripts have quite simple cisacting elements: The $\mathrm{K} 10$ transport localization signal (TLS) and the gurken localization signal (GLS) are short stem-loops of only 44 and 65 nucleotides (nt), both of which are necessary and sufficient for RNA localization to the apical cytoplasm of the embryo and to the anterior of the oocyte (Cohen et al. 2005; Van De Bor et al. 2005).

The minimal localization signals of pair-rule transcripts are more complex. The hairy localization element (HLE) is $121 \mathrm{nt}$ long and comprises two partially redundant stem-loop structures (SL1 and SL2). The ftz localization element (FLE) has a similar organization (Bullock et al. 2003; Snee et al. 2005). An intensive analysis of the bicoid localization element (BLE) defined a 53-nt stemloop region (BLE1) that, when duplicated, is sufficient for transport from the nurse cells to the oocyte (Macdonald et al. 1993). A point mutation in BLE1 that disrupts early $b c d$ localization also inhibits apical $b c d$ accumulation in blastoderm embryos (Macdonald and Kerr 1997; Bullock and Ish-Horowicz 2001).

The localization elements mentioned above all couple mRNAs to the same dynein-mediated transport machinery and share common structural features, but they lack any obvious sequence similarities (dos Santos et al. 2008). Furthermore, an attempt to find common recognition factors in embryos and oocyte extracts using the $f t z$ LE and the $b c d$ BLE1 elements showed that the two elements only partially compete for the same RNA-binding factors in a gel shift assay, leading to the conclusion that "the general strategy for recognition of localization signals is conserved, even though the recognition machinery does not appear to be highly conserved" (Snee et al. 2005).

The only components that are known to be required for dynein-dependent mRNA transport from the nurse cells into the oocyte and for apical localization in the early embryo are the dynactin complex, Bicaudal-D (Bic-D), and Egalitarian (Egl) (Bullock and Ish-Horowicz 2001; Wilkie and Davis 2001). Dynein actively transports cargoes toward minus ends of MTs and associates with the large multiprotein dynactin complex, which facilitates dynein's processivity and interaction with its cargoes. Egl and Bic-D also associate with the dynein motor complex. Mammalian Bic-D2 copurifies with dynein and dynactin and binds to the dynamitin subunit of dynactin in a yeast two-hybrid assay, while Egl binds to the dynein light chain (Dlc) (Hoogenraad et al. 2001; Navarro et al. 2004). Finally, Egl and Bic-D are part of the same complex, since they coimmunoprecipitate and colocalize with each other at all stages of oogenesis and interact with each other and dynein genetically (Mach and Lehmann 1997; Navarro et al. 2004).

When localizing RNAs are injected into blastoderm embryos, they recruit Bic-D and Egl within 1-2 min, and this occurs in the absence of MTs and before apical transport has started (Bullock and Ish-Horowicz 2001). Moreover, depletion of Bic-D inhibits the recruitment of dynein to the RNA (Bullock et al. 2006). This suggests that only transcripts that recruit Egl/Bic-D can be transported apically, and that Bic-D and Egl function to somehow link localizing mRNAs to the dynein/dynactin complex (Bullock and Ish-Horowicz 2001).

\section{Egalitarian is the elusive RNA-binding protein for dynein-dependent mRNA localization}

In this issue of Genes \& Development, Dienstbier et al. (2009) present evidence that Egl is the missing link between localizing mRNAs and the dynein motor complex. Egl is capable of selectively recognizing RNA localization signals for dynein-mediated transport and directly interacts with the cargo-binding domain of Bic-D.

To identify components of RNA recognition machinery, Dienstbier et al. (2009) used a technique in which the RNA of interest is fused to an RNA aptamer that has been selected to bind streptavidin with high affinity, and the resulting fusion RNA is attached to a streptavidin resin 
and incubated with a protein extract (Srisawat and Engelke 2001). As shown previously, the Dhc, Bic-D, and Egl specifically associate with the hairy localization element using this assay (Bullock et al. 2006). Dienstbier et al. (2009) chose to use minimal localization signals (44-63 nt) of TLS (K10), GLS (grk), SL1 (h), and ILS (I factor retrotransposon). As a control, they also used the same RNAs containing subtle mutations that prevent apical localization of the RNAs, but have little effect on their predicted secondary structures.

Surprisingly, the only proteins that were specifically and stably enriched on all tested localization elements were Egalitarian and Bicaudal-D. Bic-D is an $\alpha$-helical coil-coil protein, whereas Egl is a $120-\mathrm{kDa}$ protein without any pronounced domain structure. Since neither contains any obvious RNA-binding domains, it was not clear whether either or both proteins could directly contact RNA. To clarify this question, Dienstbier et al. (2009) performed a series of in vitro RNA-binding experiments. Using the aptamer fusion approach, they showed that full-length Bic-D translated in a rabbit reticulocyte lysate extract did not bind to RNA, nor did various truncated forms of the protein. In contrast, full-length Egl associated with RNA, and could discriminate between TLS or ILS RNAs and their nonlocalizing mutants. This result suggests that Egl, not Bic-D, confers RNA-binding activity to the Egl/Bic-D complex (Fig. 1A).

The use of rabbit reticulocyte lysate in the RNAbinding assay cannot exclude the possibility that the Egl-RNA interaction is indirect and is mediated by factors in the lysate. To rule out this possibility, Dienstbier et al. (2009) expressed recombinant Egl in yeast, either alone or as a heteromeric complex with Bic-D. Gel retardation assays showed that the Egl-Bic-D complex has a similar RNA-binding specificity as in vitro translated Egl (three times higher affinity for TLS than for the nonlocalizing mutants). Recombinant Egl alone bound RNA nonspecifically. However, this may be because it does not fold correctly in the absence of Bic-D or because the interaction with Bic-D (which might be present in the rabbit reticulocyte lysate) improves its RNA-binding specificity. The relatively low specificity of Egl's RNA binding in vitro is in good agreement with the finding that only twofold overexpression of Egl in blastoderm embryos leads to apical accumulation of otherwise nonlocalizing mRNAs (Bullock et al. 2006). Thus, Egalitarian appears to be the elusive RNA-binding protein that links localized RNAs to dynein.

\section{Bic-D and dynein complex organization}

Whereas Egl has been found so far only in invertebrates and has been implicated only in mRNA transport, Bic-D has a more broad spectrum of activities. It is conserved from flies to mammals and, beside mRNA localization, is involved in the dynein-dependent transport of Golgi vesicles and lipid droplets (Matanis et al. 2002; Larsen et al. 2008). Recruitment of Bic-D to vesicles is mediated by a direct interaction between its C-terminal domain (CTD) and the membrane-linked GTPase Rab6 (Matanis et al. 2002). Using the yeast two-hybrid technique and a series of in vitro pull-downs, Dienstbier et al. (2009) demonstrate that the $\mathrm{N}$-terminal region of Egl also binds directly to the CTD of Bic-D. Moreover, previously described loss-of-function point mutations in Egl and Bic-D inhibit the interaction between these two proteins. This raises the question of whether Bic-D and Egl always function together or whether they only form a complex to transport RNAs.

Using size exclusion chromatography, Dienstbier et al. (2009) observed that most Egl in embryonic extracts was associated with RNA-containing complexes, whereas only a small proportion of Bic-D was found in this fraction. Moreover, expressing extra Egl in the embryo inhibited the apical movement of lipid droplets rather than stimulating it. This suggests that Egl is specific for
A

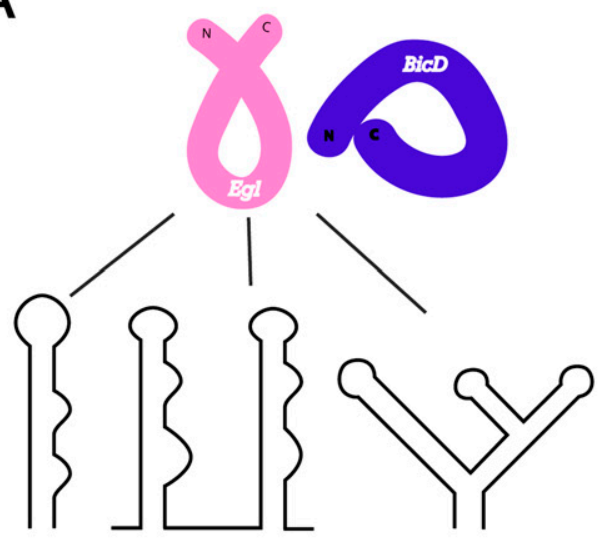

B

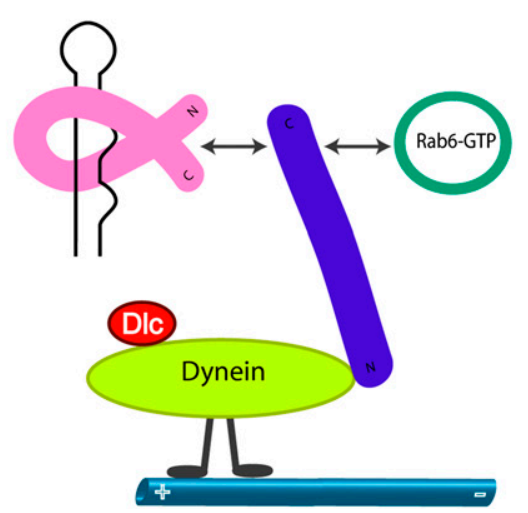

C

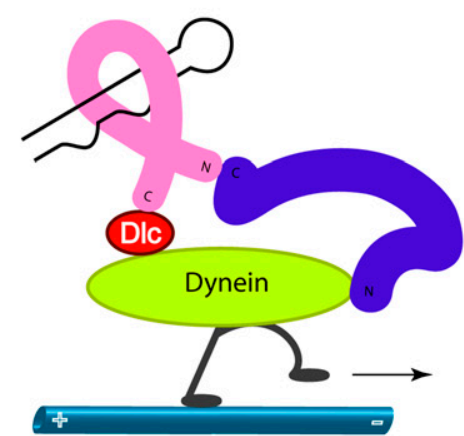

Figure 1. A model for the recruitment of RNA to dynein by Egl. $(A)$ Egl recognizes a variety of apical RNA localization signals that lack any obvious sequence similarity. (B) Egl binds to the CTD of Bic-D to release its autoinhibitory effect on the N-terminal domain, which can then interact with dynein. $(C)$ Both Bic-D/dynein and Egl/Dlc interactions stimulate dynein motor activity, leading to RNA transport toward MT minus ends. 
RNA transport and competes with other cargoes for binding to the Bic-D CTD. Consistent with this, a point mutation in the Bic-D CTD that disrupts its interaction with Egl also abolishes the CTD-Rab6 interaction. These results lead to the proposal that Bic-D mediates various types of transport through the mutually exclusive association of the CTD with different adaptor proteins.

Studies in mammalian cells have shown that the $\mathrm{N}$-terminal domain of Bic-D interacts with dynein (Hoogenraad et al. 2001). When fused to mitochondria or peroxisome anchoring sequences, this domain is sufficient to induce MT minus end-directed transport of these heterologous cargoes (Hoogenraad et al. 2003). Fusions of these anchoring sequences to full-length Bic-D are much less active in recruiting dynein, however. Furthermore, the CTDs of both mammalian and Drosophila Bic-D bind to their corresponding $\mathrm{N}$-terminal domains, suggesting that the CTD plays an autoinhibitory function by holding the protein in an inactive conformation in the absence of cargo. When CTD is bound to a cargo, this releases the $\mathrm{N}$ terminus to interact with dynein, thereby stimulating transport (Hoogenraad et al. 2001, 2003).

Dienstbier et al. (2009) were interested to see what happened if one links an RNA-binding sequence to fulllength Bic-D or to Bic-D lacking the CTD (Bic-D $\Delta$ CTD). Would it still be able to recruit dynein to mediate RNA localization? To do so, they expressed fusions between Bic-D and the MS2 bacteriophage coat protein (CP), which binds with high affinity to a short RNA hairpin. They then analyzed whether the fusion proteins could mediate the apical localization in the blastoderm embryo of an RNA containing six tandem copies of this RNA sequence (MS2 RNA). Their results fit very well to the proposed model for Bic-D regulation (Fig. 1B). MS2 RNA was localized very efficiently to the apical cytoplasm in the presence of Bic-D $\Delta$ CTD:CP, but full-length Bic-D:CP was much less active. The localization was dyneindependent and mirrored the behavior of endogenous apical transcripts.

If Bic-D $\Delta$ CTD:CP brings RNA directly to the dynein it should be independent of RNA adaptor Egl and endogenous Bic-D. However, blocking endogenous full-length Bic-D or Egl by antibody injection prevented the apical localization of MS2 RNA by Bic-D $\Delta$ CTD:CP. This suggests that the interaction between the $\mathrm{N}$-terminal part of Bic-D and the dynein complex is insufficient to promote RNA transport, and that this also requires Bic-D that contains the CTD, as well as Egl. How, then, does the MS2 RNA become localized apically? Dienstbier et al. (2009) reasoned that since Bic-D $\Delta$ CTD was able to interact with full-length Bic-D in a yeast two-hybrid assay, BicD $\Delta$ CTD:CP could recruit endogenous Bic-D and Egl, which are both necessary to form an active transport complex.

\section{How is dynein regulated?}

By demonstrating that the Bic-D-Egl complex recognizes RNAs through Egl and then couples them to dynein through Egl-Dlc and Bic-D-dynein interactions, the results of Dienstbier et al. (2009) provide the first com- plete link between localizing mRNAs and the dynein motor complex. Bic-D and Egl must do more than just bring the RNA to dynein, however, because both fulllength Bic-D and Egl are crucial for the formation of an active localization complex when RNA is linked to dynein through Bic-D $\Delta$ CTD:CP. This suggests that Bic-D and Egl also somehow regulate dynein activity. Consistent with this, a point mutation in Egl that inhibits its interaction with Dlc, but not with Bic-D or RNA, severely compromises RNA transport (Navarro et al. 2004; Bullock et al. 2006). Thus, it seems likely that Egl serves two functions: It links RNA to dynein through its interaction with Bic-D, and it activates dynein's motor activity through its interaction with Dlc (Fig. 1C) The CTD of mammalian Bic-D also has been shown to interact with the dynamitin subunit of dynactin, providing a third possible link between the Bic-D/Egl/RNA complex and the dynein/dynactin motor complex, which may play an additional role in stimulating transport (Hoogenraad et al. 2001).

Egl is only required for RNA localization, raising the question of how dynein is activated in other forms of BicD-dependent transport. In this context, it is intriguing that another Bic-D-binding protein, Rab6, can also interact with the Dlc (Wanschers et al. 2008). Thus, this may be a common mechanism by which Bic-D cargo adaptors activate dynein motility.

The analysis of Dienstbier et al. (2009) highlights the power of the Bic-D/Egl/RNA transport pathway as a paradigm for understanding how dynein is regulated. The RNA cargoes are very well-defined and can be synthesized in vitro; most if not all of the protein components have been identified and can be expressed in modified forms in vivo, and it is possible to track the motility of RNA particles with high temporal resolution. These advantages make this an ideal system for gaining further insights into how dynein is regulated and how its activity is coordinated with that of other motors.

\section{RNA recognition by Egl}

The discovery that Egl recognizes a variety of RNA localization signals raises several interesting questions. First, Egl seems to represent a new class of RNA-binding protein. Its RNA-binding domain spans $>800$ amino acids, and it lacks any well-characterized RNA-binding motifs. Indeed, its only region of homology outside of insects is a central region of $\sim 100$ amino acids that resembles the prokaryotic $3^{\prime}-5^{\prime}$ exonuclease RNase D (Mach and Lehmann 1997). Mutation of five conserved residues that are required for the catalytic activity of RNase D has no effect on Egl function in vivo or its RNA-binding activity in vitro, however, indicating that Egl is unlikely to be an RNase. Nevertheless, this region is essential for RNA binding in vitro, and the recent crystal structure of RNase D may provide clues as to how Egl recognizes RNA (Zuo et al. 2005).

Second, Egl binds a variety of RNA localization elements that do not have any apparent sequence similarity. Analyses of the K10 and wingless localization elements 
indicate that the $\mathrm{Egl}$ recognizes the distal doublestranded stems of these RNAs, rather than the singlestranded stem-loop structures (Cohen et al. 2005; dos Santos et al. 2008). The bases in the major groove of dsRNA are not normally accessible for protein binding, and this suggests that the specificity of Egl binding is determined by some cryptic feature of the minor groove of these RNAs or an unusual tertiary structure of the RNA helix. It is not obvious, however, what distinguishes RNA stem-loop structures that function as localization elements from those that do not. This enigmatic but fascinating question can therefore probably only be solved by analyzing the structures of the localization signals and corresponding nonlocalizing mutants.

Although Egl and Bic-D provide a simple way to link many different mRNAs to dynein, other RNA signals must also be able to regulate the behavior of the dynein transport complex. For example, while both grk and bcd mRNAs require Egl and Bic-D for their transport from the nurse cells into the oocyte, they show different localizations within the oocyte, both of which are dynein dependent (MacDougall et al. 2003; Weil et al. 2006). This suggests that other localization elements bound by distinct RNA-binding proteins modify the activity of dynein to direct the dorsal/anterior and anterior localization of grk and $b c d$ mRNA, respectively. In addition, dynein acts as a static anchor to hold grk and pair-rule mRNAs in place once they have been localized, and this process is disrupted by antibodies against the dynein heavy chain and the dynein intermediate chain, but not by antibodies directed against Bic-D or Egl (Delanoue and Davis 2005; Delanoue et al. 2007). Dynein must therefore somehow remain attached to RNA when its motor activity is turned off, and this may require novel RNA-binding proteins that have yet to be identified. Thus, the study of dynein-dependent RNA transport seems likely to continue to produce insights into the complex question of how motor proteins are regulated in vivo.

\section{References}

Bastock R, St Johnston D. 2008. Drosophila oogenesis. Curr Biol 18: R1082-R1087. doi: 10.1016/j.cub.2008.09.011.

Bohl F, Kruse C, Frank A, Ferring D, Jansen RP. 2000. She2p, a novel RNA-binding protein tethers ASH1 mRNA to the Myo4p myosin motor via She3p. EMBO J 19: 5514-5524.

Brendza RP, Serbus LR, Duffy JB, Saxton WM. 2000. A function for kinesin I in the posterior transport of oskar mRNA and Staufen protein. Science 289: 2120-2122.

Bullock SL, Ish-Horowicz D. 2001. Conserved signals and machinery for RNA transport in Drosophila oogenesis and embryogenesis. Nature 414: 611-616.

Bullock SL, Zicha D, Ish-Horowicz D. 2003. The Drosophila hairy RNA localization signal modulates the kinetics of cytoplasmic mRNA transport. EMBO J 22: 2484-2494.

Bullock SL, Stauber M, Prell A, Hughes JR, Ish-Horowicz D, Schmidt-Ott U. 2004. Differential cytoplasmic mRNA localization adjusts pair-rule transcription factor activity to cytoarchitecture in dipteran evolution. Development 131: 4251-4261.

Bullock SL, Nicol A, Gross SP, Zicha D. 2006. Guidance of bidirectional motor complexes by mRNA cargoes through control of dynein number and activity. Curr Biol 16: 14471452.

Caceres L, Nilson LA. 2005. Production of gurken in the nurse cells is sufficient for axis determination in the Drosophila oocyte. Development 132: 2345-2353.

Clark A, Meignin C, Davis I. 2007. A Dynein-dependent shortcut rapidly delivers axis determination transcripts into the Drosophila oocyte. Development 134: 1955-1965.

Cohen RS, Zhang S, Dollar GL. 2005. The positional, structural, and sequence requirements of the Drosophila TLS RNA localization element. RNA 11: 1017-1029.

Davis I, Ish-Horowicz D. 1991. Apical localization of pair-rule transcripts requires 3 ' sequences and limits protein diffusion in the Drosophila blastoderm embryo. Cell 67: 927-940.

Delanoue R, Davis I. 2005. Dynein anchors its mRNA cargo after apical transport in the Drosophila blastoderm embryo. Cell 122: 97-106.

Delanoue R, Herpers B, Soetaert J, Davis I, Rabouille C. 2007. Drosophila Squid/hnRNP helps Dynein switch from a gurken mRNA transport motor to an ultrastructural static anchor in sponge bodies. Dev Cell 13: 523-538.

Dienstbier M, Boehl F, Li X, Bullock SL. 2009. Egalitarian is a selective RNA-binding protein linking mRNA localization signals to the dynein motor. Genes Dev (this issue). doi: $10.1101 /$ gad.531009.

dos Santos G, Simmonds AJ, Krause HM. 2008. A stem-loop structure in the wingless transcript defines a consensus motif for apical RNA transport. Development 135: 133-143.

Dubowy J, Macdonald PM. 1998. Localization of mRNAs to the oocyte is common in Drosophila ovaries. Mech Dev 70: 193-195.

Forrest KM, Gavis ER. 2003. Live imaging of endogenous RNA reveals a diffusion and entrapment mechanism for nanos mRNA localization in Drosophila. Curr Biol 13: $1159-1168$.

Gonsalvez, G.B., Urbinati, C.R., and Long, R.M. 2005. RNA localization in yeast: Moving towards a mechanism. Biol Cell 97: 75-86.

Hoogenraad CC, Akhmanova A, Howell SA, Dortland BR, De Zeeuw CI, Willemsen R, Visser P, Grosveld F, Galjart N. 2001. Mammalian Golgi-associated Bicaudal-D2 functions in the dynein-dynactin pathway by interacting with these complexes. EMBO J 20: 4041-4054.

Hoogenraad CC, Wulf P, Schiefermeier N, Stepanova T, Galjart N, Small JV, Grosveld F, de Zeeuw CI, Akhmanova A. 2003. Bicaudal D induces selective dynein-mediated microtubule minus end-directed transport. EMBO I 22: 6004-6015.

Horne-Badovinac S, Bilder D. 2008. Dynein regulates epithelial polarity and the apical localization of stardust A mRNA. PLoS Genet 4: e8. doi: 10.1371/journal.pgen.0040008.

Hughes JR, Bullock SL, Ish-Horowicz D. 2004. Inscuteable mRNA localization is dynein-dependent and regulates apicobasal polarity and spindle length in Drosophila neuroblasts. Curr Biol 14: 1950-1956.

Jenny, A, Hachet, O, Zavorszky, P, Cyrklaff, A, Weston, MD, Johnston, DS, Erdelyi, M, and Ephrussi, A. 2006. A translationindependent role of oskar RNA in early Drosophila oogenesis. Development 133: 2827-2833.

Johnstone O, Lasko P. 2001. Translational regulation and RNA localization in Drosophila oocytes and embryos. Annu Rev Genet 35: 365-406.

King, M.L., Messitt, T.J., and Mowry, K.L. 2005. Putting RNAs in the right place at the right time: RNA localization in the frog oocyte. Biol Cell 97: 19-33.

Larsen KS, Xu J, Cermelli S, Shu Z, Gross SP. 2008. BicaudalD actively regulates microtubule motor activity in lipid droplet 
transport. PLoS One 3: e3763. doi: 10.1371/journal.pohne. 0003763.

Lecuyer E, Yoshida $\mathrm{H}$, Parthasarathy N, Alm C, Babak T, Cerovina T, Hughes TR, Tomancak P, Krause HM. 2007. Global analysis of mRNA localization reveals a prominent role in organizing cellular architecture and function. Cell 131: 174-187.

Li Z, Wang L, Hays TS, Cai Y. 2008. Dynein-mediated apical localization of crumbs transcripts is required for Crumbs activity in epithelial polarity. J Cell Biol 180: 31-38.

Lin AC, Holt CE. 2007. Local translation and directional steering in axons. $E M B O / 26: 3729-3736$.

Macdonald PM, Kerr K. 1997. Redundant RNA recognition events in bicoid mRNA localization. RNA 3: 1413-1420.

Macdonald PM, Kerr K, Smith JL, Leask A. 1993. RNA regulatory element BLE1 directs the early steps of bicoid mRNA localization. Development 118: 1233-1243.

MacDougall N, Clark A, MacDougall E, Davis I. 2003. Drosophila gurken $(\mathrm{TGF} \alpha)$ mRNA localizes as particles that move within the oocyte in two dynein-dependent steps. Dev Cell 4: 307-319.

Mach JM, Lehmann R. 1997. An Egalitarian-BicaudalD complex is essential for oocyte specification and axis determination in Drosophila. Genes \& Dev 11: 423-435.

Martin KC, Ephrussi A. 2009. mRNA localization: Gene expression in the spatial dimension. Cell 136: 719-730.

Martin KC, Zukin RS. 2006. RNA trafficking and local protein synthesis in dendrites: An overview. I Neurosci 26: 7131-7134.

Matanis T, Akhmanova A, Wulf P, Del Nery E, Weide T, Stepanova T, Galjart N, Grosveld F, Goud B, De Zeeuw CI, et al. 2002. Bicaudal-D regulates COPI-independent Golgi-ER transport by recruiting the dynein-dynactin motor complex. Nat Cell Biol 4: 986-992.

Mische S, Li M, Serr M, Hays TS. 2007. Direct observation of regulated ribonucleoprotein transport across the nurse cell/ oocyte boundary. Mol Biol Cell 18: 2254-2263.

Navarro C, Puthalakath H, Adams JM, Strasser A, Lehmann R. 2004. Egalitarian binds dynein light chain to establish oocyte polarity and maintain oocyte fate. Nat Cell Biol 6: 427-435.

Paquin N, Chartrand P. 2008. Local regulation of mRNA translation: New insights from the bud. Trends Cell Biol 18: 105-111.

Simmonds AJ, dosSantos G, Livne-Bar I, Krause HM. 2001. Apical localization of wingless transcripts is required for wingless signaling. Cell 105: 197-207.

Snee MJ, Arn EA, Bullock SL, Macdonald PM. 2005. Recognition of the bcd mRNA localization signal in Drosophila embryos and ovaries. Mol Cell Biol 25: 1501-1510.

Srisawat C, Engelke DR. 2001. Streptavidin aptamers: Affinity tags for the study of RNAs and ribonucleoproteins. RNA 7: 632-641.

St Johnston D. 2005. Moving messages: The intracellular localization of mRNAs. Nat Rev Mol Cell Biol 6: 363-375.

St Johnston D, Driever W, Berleth T, Richstein S, NussleinVolhard C. 1989. Multiple steps in the localization of bicoid RNA to the anterior pole of the Drosophila oocyte. Development 107: 13-19.

Van De Bor V, Hartswood E, Jones C, Finnegan D, Davis I. 2005. gurken and the I factor retrotransposon RNAs share common localization signals and machinery. Dev Cell 9: 51-62.

Wanschers B, van de Vorstenbosch R, Wijers M, Wieringa B, King SM, Fransen J. 2008. Rab6 family proteins interact with the dynein light chain protein DYNLRB1. Cell Motil Cytoskeleton 65: 183-196.

Weil TT, Forrest KM, Gavis ER. 2006. Localization of bicoid mRNA in late oocytes is maintained by continual active transport. Dev Cell 11: 251-262.
Wilkie GS, Davis I. 2001. Drosophila wingless and pair-rule transcripts localize apically by dynein-mediated transport of RNA particles. Cell 105: 209-219.

Zimyanin VL, Belaya K, Pecreaux J, Gilchrist MJ, Clark A, Davis I, St Johnston D. 2008. In vivo imaging of oskar mRNA transport reveals the mechanism of posterior localization. Cell 134: 843-853.

Zuo Y, Wang Y, Malhotra A. 2005. Crystal structure of Escherichia coli RNase D, an exoribonuclease involved in structured RNA processing. Structure 13: 973-984. 


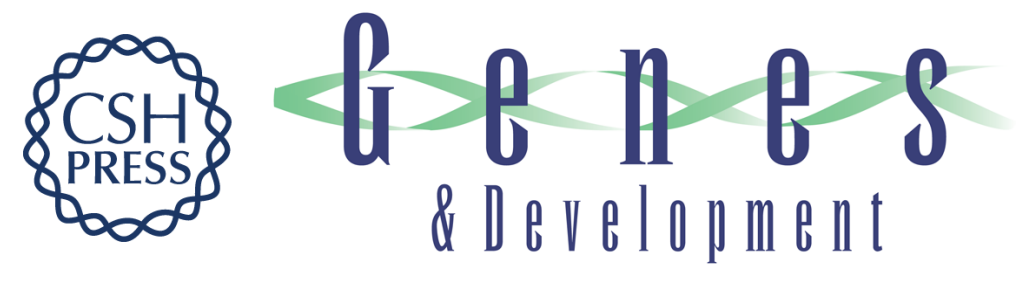

\section{Egalitarian recruitment of localized mRNAs}

Dmitry Nashchekin and Daniel St Johnston

Genes Dev. 2009, 23:

Access the most recent version at doi:10.1101/gad.1821509

\section{Related Content Egalitarian is a selective RNA-binding protein linking mRNA localization signals to the dynein motor \\ Martin Dienstbier, Florian Boehl, Xuan Li, et al. \\ Genes Dev. July , 2009 23: 1546-1558 \\ References This article cites 50 articles, 20 of which can be accessed free at: \\ http://genesdev.cshlp.org/content/23/13/1475.full.html\#ref-list-1 \\ Articles cited in: \\ http://genesdev.cshlp.org/content/23/13/1475.full.html\#related-urls \\ License \\ Email Alerting \\ Service \\ Receive free email alerts when new articles cite this article - sign up in the box at the top right corner of the article or click here.}

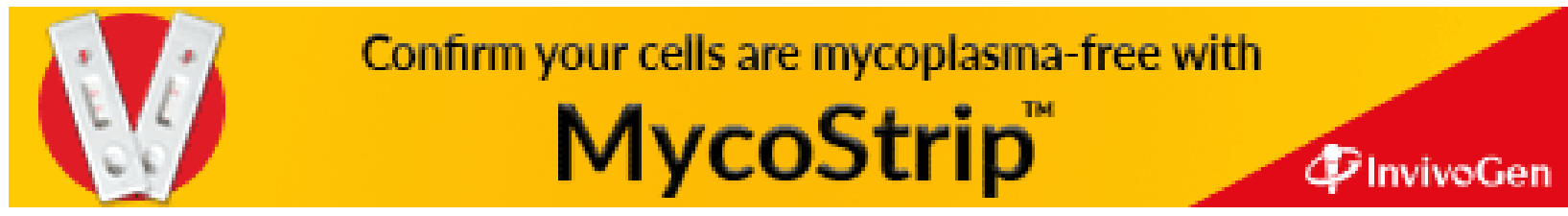

